\title{
Indium In 111-labeled Macroaggregated Albumin
}

National Cancer Institute

\section{Source}

National Cancer Institute. Indium In 111-labeled Macroaggregated Albumin. NCI

Thesaurus. Code C116856.

A radiopharmaceutical formulation containing human, serum albumin (HSA) labeled with indium In 111 (In-111) in macroaggregates (MAA) with potential usage as a diagnostic imaging agent. Upon injection of indium In 111-labeled macroaggregated albumin and subsequent imaging, biodistribution patterns can be assessed and the expected distribution of agents with similar particle sizes may be predicted. 